\title{
Local extinction and reignition mechanism in a turbulent lifted flame: a direct numerical simulation study
}

\author{
Shahram Karami ${ }^{\mathrm{a}}$, Mohsen Talei ${ }^{\mathrm{c}}$, Evatt R. Hawkes ${ }^{\mathrm{a}, \mathrm{b}}$, \\ Jacqueline H. Chen ${ }^{\mathrm{d}}$ \\ ${ }^{\text {a }}$ School of Photovoltaic and Renewable Energy Engineering, \\ University of New South Wales, Sydney, 2052, Australia \\ ${ }^{\mathrm{b}}$ School of Mechanical and Manufacturing Engineering, \\ University of New South Wales, Sydney, 2052, Australia \\ ${ }^{\mathrm{c}}$ School of Mechanical Engineering, \\ University of Melbourne, Melbourne, 3010, Australia \\ d Combustion Research Facility, \\ Sandia National Laboratories, Livermore, California, CA 94551, United States of America
}

Corresponding author:

Evatt R. Hawkes

School of Photovoltaic and Renewable Energy Engineering

School of Mechanical and Manufacturing Engineering

University of New South Wales

Sydney, 2052, Australia

Phone: +61 (2) 93854602

Fax: +61 (2) 93857762

Email: evatt.hawkes@unsw.edu.au

Preferred colloquium topic area:

Turbulent Flames

Total length of paper:

$(6.75$ pages $* 900)=6075(\operatorname{method} 2)$

Submitted to the Proceedings of the Combustion Institute 


\begin{abstract}
Local extinction and reignition is studied in a direct numerical simulation (DNS) dataset of a turbulent lifted flame [1]. Extinction holes are identified as regions on the stoichiometric surface which have a product mass fraction less than a critical value. Using this criterion, thirty individual holes are identified and tracked in time. It is observed that large outwardly pushing structures caused compressive strain rates normal to the mixture-fraction iso-surface. These high strain rates caused high dissipation rates and the initiation of the extinction process, leading to the initial creation of holes. Extinction, i.e. hole growth, then occurs in two phases. In the first phase, the edge-propagation velocity is initially negative and the fluid dynamic tangential strain rate on the hole surface is positive, leading to rapid hole growth. Subsequently, in the second phase, local compressive strain rates at the flame edge relax, and the edge-flame propagation velocity switches to positive. However, in this second phase, the hole continues to expand because of positive tangential strain rate on the hole's surface, which dominates over the healing effect of positive edge-flame propagation velocities. When reignition starts, the edge-propagation velocity is mainly affected by the product-mass fraction displacement speed and shows a dependency on curvature and scalar dissipation rate, similar to what is expected in edge-flame propagation. An analysis of thermal diffusion on the unburned portion of the mixture-fraction iso-surface shows that the edge-flame propagation mechanism dominates turbulent engulfment during reignition in this study.
\end{abstract}

Keywords: Lifted flame; Local extinction; Reignition; Edge flame; DNS 


\title{
Local extinction and reignition mechanism in a turbulent lifted flame: a direct numerical simulation study
}

\author{
Shahram Karami ${ }^{\mathrm{a}}$, Mohsen Talei ${ }^{\mathrm{b}}$, Evatt R. Hawkes ${ }^{\mathrm{a}, \mathrm{c}, *}$, Jacqueline H. Chen ${ }^{\mathrm{d}}$ \\ ${ }^{a}$ School of Photovoltaic and Renewable Energy Engineering, The University of New South \\ Wales, Sydney, NSW 2052, Australia \\ ${ }^{b}$ School of Mechanical Engineering, University of Melbourne, Melbourne, 3010 Australia \\ ${ }^{c}$ School of Mechanical and Manufacturing Engineering, The University of New South Wales, \\ Sydney, NSW 2052, Australia \\ ${ }^{d}$ Combustion Research Facility, Sandia National Laboratories, Livermore, California, CA \\ 94551, United States of America
}

\begin{abstract}
Local extinction and reignition is studied in a direct numerical simulation (DNS) dataset of a turbulent lifted flame [1]. Extinction holes are identified as regions on the stoichiometric surface which have a product mass fraction less than a critical value. Using this criterion, thirty individual holes are identified and tracked in time. It is observed that large outwardly pushing structures caused compressive strain rates normal to the mixture-fraction iso-surface. These high strain rates caused high dissipation rates and the initiation of the extinction process, leading to the initial creation of holes. Extinction, i.e. hole growth, then occurs in two phases. In the first phase, the edge-propagation velocity is initially negative and the fluid dynamic tangential strain rate on the hole surface is positive, leading to rapid hole growth. Subsequently, in the second phase, local compressive strain rates at the flame edge relax, and the edge-flame propagation velocity switches to positive. However, in this second phase, the hole continues to expand because of positive tangential strain rate on the hole's surface, which dominates over the healing effect of positive edge-flame propagation velocities. When reignition starts, the edge-propagation velocity is mainly affected by the product-mass fraction displacement speed and shows a dependency on curvature and scalar dissi-
\end{abstract}

\footnotetext{
*Corresponding author

Email address: evatt.hawkes@unsw.edu.au (Evatt R. Hawkes)
} 
pation rate, similar to what is expected in edge-flame propagation. An analysis of thermal diffusion on the unburned portion of the mixture-fraction iso-surface shows that the edge-flame propagation mechanism dominates turbulent engulfment during reignition in this study.

Keywords:

Lifted flame, Local extinction, Reignition, Edge flame, DNS 


\section{Introduction}

The rate of mixing between fuel and air plays an important role in the flame stability and pollutant emissions for non-premixed flames. High mixing rates are generally desirable to decrease the size of a combustor and to enhance premixing leading to lower pollutant emissions. When mixing rates exceed a critical level, local extinctions can occur, appearing as holes in the reacting sheet, i.e. on the mixture fraction iso-surface. Extinction may lead to increased harmful emissions of unburned or partially burned fuel, and if pervasive, to flame blowout which is obviously not desirable. As a result, local extinction and reignition have received significant attention in the literature.

Local extinctions have been observed in experimental studies of laminar nonpremixed counterflows and jets [2-4], turbulent piloted jet flames [5, 6], turbulent rim

stabilised flames [7], and turbulent lifted flames [8,9]. These local extinctions were shown to be correlated with local strain rate, scalar dissipation rates, and/or vortical flow structures $[2,3,7,8]$. However, these studies are restricted to measuring a limited number of parameters, such as scalar dissipation rate, velocity field and some of the species concentrations, and usually to two-dimensional measurements. A detailed understanding of extinction and reignition mechanisms requires access to parameters such as strain rates, scalar dissipation rate, edge-propagation velocity, curvature, etc., which would be challenging to provide experimentally.

Local extinction has been also a topic of numerical studies. A number of studies reported the importance of the scalar dissipation rate on the local extinction [10-13]. For instance, in a study of local extinction in a laminar triple flame interacting with a pair of vortices, Favier and Vervisch [10], showed that the scalar dissipation rate is the controlling parameter. Furthermore, they observed that the scalar dissipation rate of growing holes is lower than which initiates the local extinction. In a DNS a study of piloted flame, Pantano [11] also observed that extinctions are encountered in the region with a scalar dissipation higher than the laminar extinction limit. In some other numerical studies $[14,15]$, strain rates were found to play an important role in local extinction. 
An extinction event can be followed by a reignition event through a number of different mechanisms. Sripakagorn et al. [12] studied extinction and reignition by performing DNS of reacting isotropic turbulence with a single-step chemistry model. They categorised reignition scenarios as independent flamelets, engulfment and edgeflame propagation. The independent flamelet scenario is a reignition mode in which incompletely extinguished regions are able to reignite in isolation without assistance of heat conduction from other regions. In the edge-flame mode, the burning regions of the mixture-fraction iso-surface propagate into the extinguished region like a partially premixed edge flame $[15,16]$. The edge-flame scenario was further investigated and modelled in a study by Pantano and Pullin [17]. Another scenario is engulfment or flame folding in which the hot surfaces are pulled towards the extinguished surface by turbulent convection. Most of the previous studies found that a combination of these scenarios govern the reignition phenomenon $[12,15,16]$.

These reviewed experimental and numerical studies have revealed some aspects of ignition and reignition. However, a comprehensive picture describing the extinction and reignition mechanisms in the context of non-premixed lifted flames has not been presented. Therefore, the main focus of this paper is to analyse extinction and reignition using direct numerical simulation (DNS). A lifted flame is selected for the study due to its relevance to practical combustion systems, and due to the key role that extinctions may play in this type of flame with respect to blow-off stability and flame dynamics. The analysis will reveal the mechanisms involved in extinction and reignition and the role of key parameters, namely the scalar dissipation rate, strain rates, curvature and normalised flame index.

\section{Method}

\subsection{Configuration and numerical methods}

The DNS database of a turbulent lifted slot-jet flame $[1,18,19]$ is used for the presented analysis. For an orientation, a brief description of key details of the DNS dataset is reported here. The jet and co-flow Mach number were 0.48 and 0.001, respectively. The Reynolds number was 5,280. Pure fuel was fed into the domain 
of cold air with a frozen turbulent velocity field with a turbulent intensity of $5 \%$. A simple one-step chemistry model was used with a modified activation energy [20] to reproduce the laminar flame speed dependency on equivalence ratio for typical hydrocarbon fuels. Further details on the numerical setup and method can be found in Ref. [1].

\subsection{Extinction holes identification}

In this study focussing on extinction and reignition, it is useful to define a concept of flame edges, which separate the burning and non-burning regions of a mixturefraction iso-surface. Following earlier studies of extinction-reignition $[11,16]$ and our previous study of stabilisation mechanism of the lifted flame [1], we select the edgeflame marker to be the intersection of a mixture-fraction iso-surface and a product mass-fraction iso-surface. The mixture fraction was taken as that having the highest laminar flame speed in a $1 \mathrm{D}$ flame $\left(Z_{m s}\right.$, which equal to 0.07 i.e. $\left.1.2 Z_{s t}\right)$, while the product mass fraction was obtained at the location of the maximum heat release rate in the same 1D flame $\left(Y_{p}=0.2\right)$.

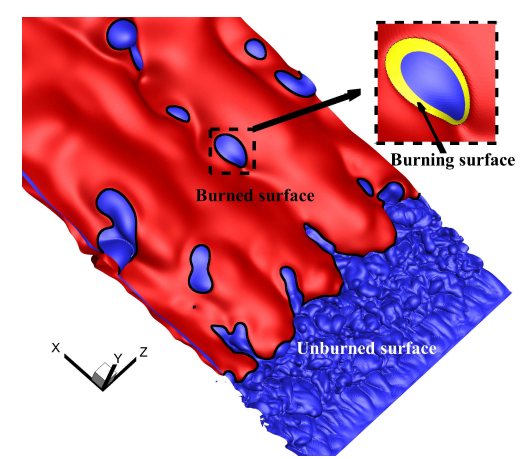

Figure 1: The schematic of the mixture-fraction iso-surface of $Z_{m s}$ (blue surface) and product mass fraction of 0.2 (red surface) with the different regions highlighted for an individual hole (Blue: unburned region, Yellow: burning region and Red: the burned region - see text for definitions).

The holes identified using this methodology were extensively visualised to understand the holes behaviour and their interaction with each other. The extinction holes may grow and shrink independently, split into multiple holes, and/or merge with neigh- 
a)
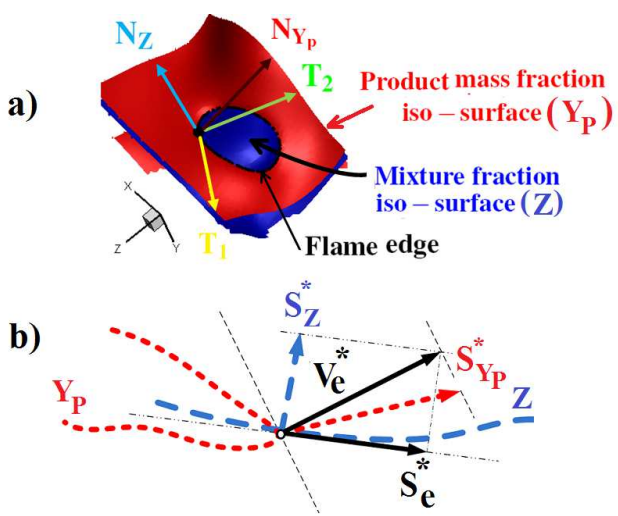

Figure 2: Schematic of a) a hole with different tangential and normal vectors specified and b) edge propagation velocities (The surfaces are the same as described in Fig. 1).

bour holes, which is consistent with the observations in previous experimental studies [8, 21, 22]. For the purpose of this study, thirty individual holes (see Fig. 1) with no splitting and merging and far from other neighbouring holes were selected to minimise the hole-hole interactions.

The edge-flame displacement speed, $S_{e}^{*}$, is defined as the motion of the flame edge relative to the flow (defined positive if towards reactants), projected into the plane of the mixture-fraction iso-surface, as shown in Fig. 2. The edge speed is given in terms of the product mass-fraction self-displacement speed $S_{d}^{*}$, the mixture-fraction self-displacement speed $S_{Z}^{*}$, and the inner product of the normal vectors to product and mixture-fraction iso-surfaces $\mathrm{k}, \boldsymbol{N}_{Y_{p}} \bullet \boldsymbol{N}_{Z}$, as [11, 16]:

$$
\begin{aligned}
S_{e}^{*} & =\frac{S_{d}^{*}-k S_{Z}^{*}}{\sqrt{1-k^{2}}}, \\
\rho_{u} S_{Z}^{*} & =\frac{1}{|\nabla Z|}\left(\frac{\partial}{\partial x_{j}}\left(\frac{\mu}{\operatorname{LePr}} \frac{\partial Z}{\partial x_{j}}\right)\right), \text { and } \\
\rho_{u} S_{d}^{*} & =\frac{1}{\left|\nabla Y_{P}\right|}\left(\dot{\omega}_{P}+\frac{\partial}{\partial x_{j}}\left(\frac{\mu}{\operatorname{LePr}} \frac{\partial Y_{P}}{\partial x_{j}}\right)\right),
\end{aligned}
$$

where $x_{j}$ presents the spatial vectors, $\mu$ the non-dimensional viscosity defined as $\mu^{*} / \operatorname{Re}_{a c}, \rho$ the density, Pr the Prandtl number, Le the Lewis number. The normalised flame index (NFI) [23] is also used here to distinguish between premixed, (when the NFI $\rightarrow+1$ ), and non-premixed, (when the NFI $\rightarrow-1$ ), combustion modes and is defined as:

$$
\mathrm{NFI}=\frac{\nabla \mathrm{Y}_{\mathrm{F}} \bullet \nabla \mathrm{Y}_{\mathrm{O}}}{\left|\nabla \mathrm{Y}_{\mathrm{F}} \bullet \nabla \mathrm{Y}_{\mathrm{O}}\right|}
$$



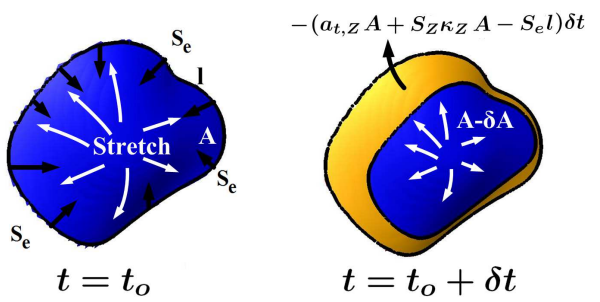

Figure 3: Schematic of a hole at two infinitessimally separated time instincts with contributions of stretch and edge-flame propagation in shrinking the unburned surface.

As discussed by Hawkes et al. [16], the stretch rate on unburned portions of the mixture-fraction iso-surface, i.e. hole area and the edge propagation velocity on the hole perimeter, are key parameters during extinction. The effect of these parameters, as shown in Fig. 3 may be formulated as,

$$
\frac{d A}{d t}=\int\left(a_{t, Z}+S_{Z} \kappa_{Z}\right) d A-\int S_{e} d l
$$

where $A$ is the hole area, $a_{t, Z}$ and $S_{Z} \kappa_{Z}$ are two components of the stretch on the mixture-fraction iso-surface, defined as,

$$
a_{t, Z}=\left(\delta_{i j}-N_{Z i} N_{Z j}\right) \frac{\partial u_{i}}{\partial x_{j}}, \text { and } \kappa_{Z}=\frac{\partial N_{Z i}}{\partial x_{i}} .
$$

Note that the stretch-related term, integrated over the hole surface area, is readily established from the flame surface density literature, e.g. [24]. The second term involving the edge-flame speed integrated over the hole perimeter represents creation of hole area by extinction (when $S_{e}$ is on average negative) or destruction of hole area by reignition (when $S_{e}$ is on average positive).

To identify the reignition mechanism, the heat diffused from and along the mixturefraction iso-surface accompanied by the normalised flame index were used [12]. The normal and tangential diffusion terms are defined as,

$$
i_{p}=\frac{\mu}{\operatorname{Pr}}\left(\frac{\partial^{2} T}{\partial t_{1}^{2}}+\frac{\partial^{2} T}{\partial t_{2}^{2}}\right), \text { and } i_{n}=\frac{\mu}{\operatorname{Pr}} \frac{\partial^{2} T}{\partial n_{3}^{2}},
$$

where $t_{1}$ and $t_{2}$ are tangential to the mixture-fraction iso-surface and $n_{3}$ is the coordinate in the normal direction. Using equations 2 and 5, the reignition scenario can be categorised, following Ref. [12]. In the flamelet scenario, the diffusion tangential to the mixture-fraction iso-surface is negligible and heat is diffused away from the surface 
in the normal direction [25]. The propagation of an edge-flame towards the unburned region will result in the heat being diffused away from the surface in the normal direction and diffused towards closing the hole in the plane of mixture-fraction iso-surface, i.e. in the tangential direction. In the engulfment scenario, reignition occurs due to interaction with a hot neighbourhood. Therefore, the heat is diffused towards the hole in the normal direction. To investigate the contribution of any of these scenarios in the

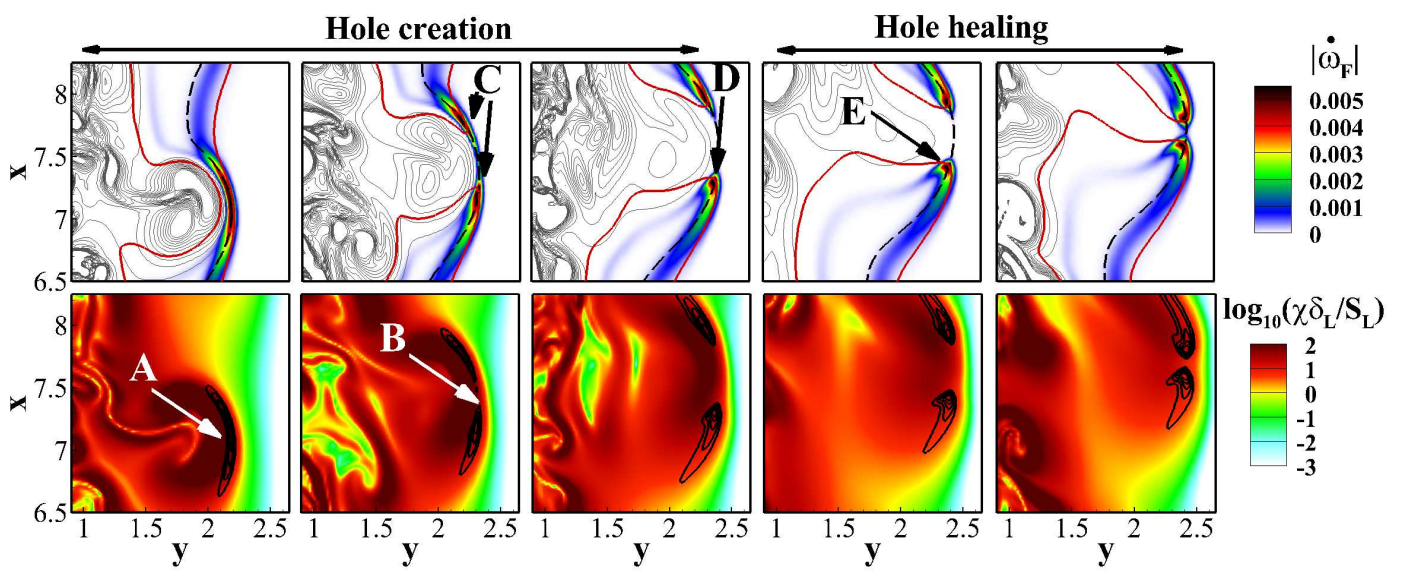

Figure 4: An instantaneous example of a common creation and healing of holes.

reignition process, it is necessary to split the extinguished surface into two different regions of the frozen mixing inert zone and the preheat zone, where the temperature is high enough to ignite the mixture. From the 1D simulation of the laminar premixed flame, the region with product mass fraction less than 0.01 is selected as the inert region and the region with product mass fraction between 0.01 and 0.2 is found to be in the preheat zone. These regions are presented in Fig. 1.

\section{Results and Discussion}

\subsection{Instantaneous results}

To demonstrate some key features of extinction and reignition, the time evolution of the reaction rate superimposed on the vorticity field and the field of scalar dissipation rate for a typical hole creation and healing is presented in Fig. 4. The fuel stream is on the left side whereas the oxidiser stream is on the right side of each sub-figure. The 
solid and dashed lines represent the product mass-fraction of 0.2 and stoichiometric mixture-fraction iso-lines, respectively. The intersection of these two lines corresponds to the hole edge. Prior to extinction, a large structure, marked by 'A', interacts with the mixture-fraction iso-surface creating a region of high scalar dissipation rate. In the second instant, a small hole is created where the scalar dissipation is still high (marked as 'B'). At this time the local edge-flame speeds are negative, and very thin, comet-shaped edge-flames are observed without a rich premixed branch at locations marked as 'C'. It can also be observed that the mixture-fraction iso-surface is bulging out as a result of interaction with a large eddy during the formation of this hole which is consistent with the observation of Lignell et al. $[15]^{1}$. At the third instant, the lower edge, marked as 'D', experiences a lower scalar dissipation rate compared with the upper edge. As a result, a triple flame with only a rich wing is observed at this point. At this point the edge-flame speed has become positive. In the next instant, reignition has commenced. The scalar dissipation rate has relaxed even further and a more distinct triple flame structure is observed at the lower edge, marked as ' $\mathrm{E}$ '.

\subsection{Conditionally averaged statistics}

To characterise the size of the hole and their transient evolution, a hole radius is calculated by integrating the distance from the hole centroid ${ }^{2}$ (based on perimeter) to the each local point on the hole perimeter with respect to the hole perimeter. The rate of change of the radius is also recorded. As an orientation, Fig. 5a shows the time evolution of the hole radius (normalised by its maximum for each hole) of three individual holes, relative to the time of initiation of each one $\left(t_{s}\right)$. As can be seen, extinction events occur over a much shorter period of time compared with the reignition events. Also, a high acceleration of the edge is observed at the early stage of

\footnotetext{
${ }^{1}$ This is also confirmed in Fig. S2 of the supplementary material, where the joint PDF of mixture fraction iso-surface curvature and scalar dissipation rates during extinction shows the high probability of mixture fraction iso-surface having a center of curvature in the fuel stream.

${ }^{2}$ The centroid of each hole is simply calculated as the average position of all the points on the perimeter of that hole.
} 

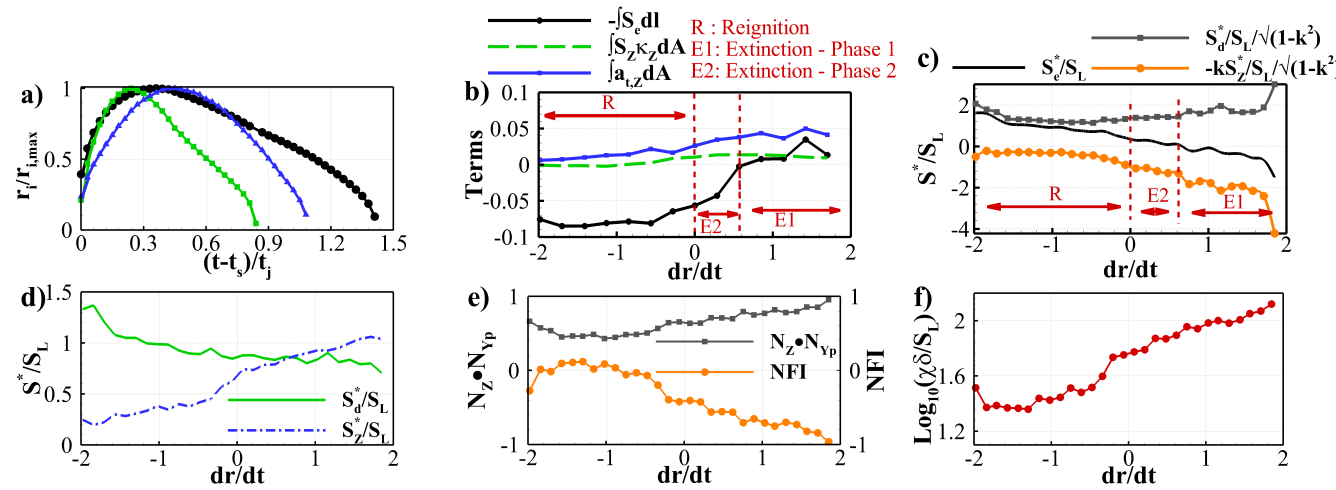

Figure 5: a) examples of time evolution of three individual holes, the mean conditioned on rate of change of radius of b) contributions in expanding unburned surface of: edge-flame propagation speed $\left(-\int S_{e} d l\right)$, components of stretch of the mixture-fraction iso-surface $\left(\int S_{Z} \kappa_{Z} d A\right.$ $\left.\& \int a_{t, Z} d A\right)$, c) density weighted edge-propagation velocity $\left(S_{e}^{*} / S_{L}\right)$, the net contribution of normalised product mass-fraction and mixture-fraction displacement speeds to $S_{e}^{*} / S_{L}, \mathrm{~d}$ ) $S_{d}^{*} / S_{L}, S_{Z}^{*} / S_{L}$, e) the inner product of the normal vectors and normalised flame index, and f) normalised scalar dissipation rate.

extinction and very late stage of reignition. Figure $5 \mathrm{~b}$ presents the various terms which govern the rate of change of the hole area as per equation 3, conditionally averaged on $d r / d t$ for the thirty holes. Positive $d r / d t$ on the right corresponds to hole growth, i.e. extinction, while negative $d r / d t$ on the left corresponds to hole shrinkage, i.e. reignition. Considering first the extinction, based on Fig. 5b it can be divided into two distinct phases. In the phase marked 'E1', the contribution of the edge-propagation velocity and both stretch terms are positive resulting in creation of holes. In the phase marked 'E2', the edge-flame propagation velocity term tends to consume unburned surfaces while the stretch terms continue to create unburned surfaces. However, the stretch terms seem in this phase to dominate the edge-propagation velocity term and therefore the holes continue to grow in phase 'E2'.

To investigate the observed behaviour for the edge-propagation velocity, Fig. 5c shows the conditionally averaged density weighted edge-propagation velocity $\left(S_{e}^{*} / S_{L}\right)$, the net contribution (using eq. 2) to $S_{e}^{*} / S_{L}$ of normalised product mass-fraction displacement speed, $S_{d}^{*} / S_{L}$ and normalised mixture-fraction displacement speed $S_{Z}^{*} / S_{L}$. As can be seen, the $S_{Z}^{*}$ component, i.e. the component due to motion of mixturefraction iso-surfaces, is the dominant contributor to the observed behaviour for $S_{e}^{*}$ 
during extinction. In both phases 'E1' and 'E2', the $S_{d}^{*}$ component competes with the $S_{Z}^{*}$ component, with the latter winning during phase 'E1' and the former winning during phase 'E2'. However, during reignition, the trend for $S_{e}^{*}$ is dominated by $S_{d}^{*} / S_{L}$. The trends shown in Fig. $5 \mathrm{c}$ are determined both by the trends for the speeds involved and by the orientations of the normal vectors. To investigate the first effect, Fig. 5d shows $S_{d}^{*} / S_{L}$ and $S_{Z}^{*} / S_{L}$ conditionally averaged on $d r / d t$ for the thirty holes. As can be seen, the mixture fraction displacement speed is the highest when the holes start to form and shows a decreasing trend during the extinction/reignition process. Comparison of Figs. 5c and d suggests that the strong negative contribution of $S_{Z}^{*}$ component to $S_{e}^{*} / S_{L}$ during the extinction event is due to the $k / \sqrt{\left(1-k^{2}\right)}$ term. This term becomes large when the normal vectors to product and mixture fraction iso-surfaces becomes aligned. Furthermore, Fig. 5 d shows that during reignition $S_{d}^{*} / S_{L}$ is larger than $S_{Z}^{*} / S_{L}$.

However, comparison of Fig. 5d) and c) shows that the influence of the $S_{Z}^{*}$ component on $S_{e}^{*}$ during the reignition phase is even smaller than suggested by the $S_{Z}^{*}$ alone, indicating that the normal vectors are not well aligned during these phase and thus the factor $k / \sqrt{\left(1-k^{2}\right)}$ is small. To confirm these alignment trends, the inner product of $N_{Y_{p}}$ and $N_{Z}$ is now shown in Fig. 5e. This figure confirms the significant contribution from the $k / \sqrt{\left(1-k^{2}\right)}$ term during the extinction event. In particular, at the early stage of extinction, the highest alignment between $N_{Y_{p}}$ and $N_{Z}$ is observed. The NFI is also presented in Fig. 5e. It suggests a predominant presence of non-premixed flame during extinction (as indicated by negative NFI values) whereas on-average neutral values are observed during reignition, suggesting that different combustion modes may simultaneously exist. However, the scalar dissipation rate gradually decreases as the holes are formed and healed. The decreasing trend of scalar dissipation rate during extinction was observed in Fig. 4 and was also reported in Ref. [10].

\subsection{Local Statistics}

In this section, the statistical response of the hole edge to different important parameters during extinction and reignition is investigated on a local basis. The data 
on all extracted flame edges are included, and they are conditioned on whether the hole is growing/extinguishing $(d r / d t>0)$ or shrinking/reigniting $(d r / d t<0)$.

\subsubsection{Extinction}
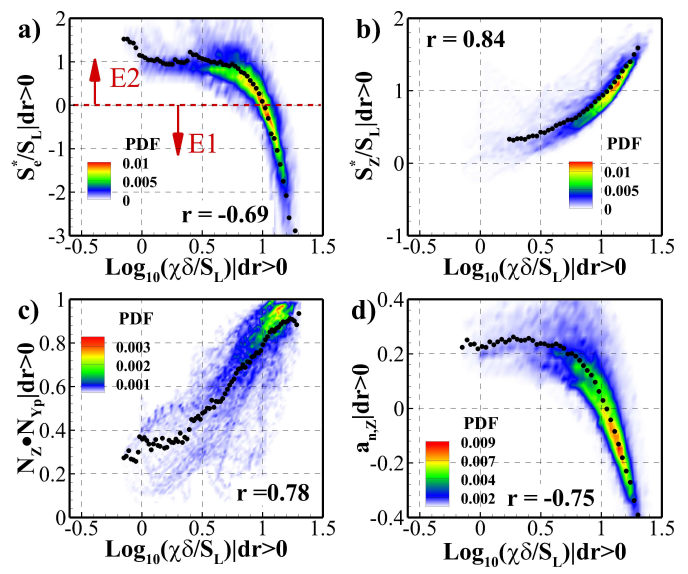

Figure 6: The joint PDF with scalar dissipation rate of: a) edge-flame propagation velocity, b) normalised mixture-fraction displacement speed, c) the inner product of the normal vectors and d) normal strain rates of the mixture-fraction iso-surfaces $a_{n, Z}$ (black circles are conditional means).

The extinguishing case is first considered. Figure 6 presents the joint probability density functions (PDFs) of a) the edge-flame propagation velocity, b) the normalised mixture-fraction displacement speed, c) the inner product of the normal vectors $N_{Y_{p}}$ and $N_{Z}$ and d) the normal strain rates of the mixture-fraction iso-surfaces $a_{n, Z}$ with the scalar dissipation rate. As shown in Fig. 6a, the edge-propagation velocity is negatively correlated with the scalar dissipation rate. The conditional mean shows a non-linear behaviour as expected during extinction $[11,26]$ and the joint PDF is narrow with low fluctuations over the conditional mean. This suggests that the scalar dissipation rate is a key controlling parameter during extinction. It is noted that the scalar dissipation rate at which the edge-propagation velocity tends to zero in the DNS is close to the laminar quenching value (see supplementary material). Figure 6b shows a strong positive correlation between the normalised mixture-fraction displacement speed and the scalar dissipation rate which is a consequence of the mixture-fraction iso-surface interacting by large eddies (shown in Fig. 4). This positive correlation 
is also consistent with the observations in Figs. 5d and e. Figure 6c also shows a positive correlation between $N_{Y_{p}} \bullet N_{Z}$ and scalar dissipation rate. In particular, a high probability of alignment between $N_{Y_{p}}$ and $N_{Z}$ is observed for high scalar dissipation rates. This is mainly related to the phase 'E1' as observed in Fig 5e. Turning now to Fig. $6 \mathrm{~d}$, it is noted that normal strain rates of the mixture-fraction iso-surfaces, $a_{n, Z}$, is negatively correlated with the scalar dissipation rate. Furthermore, high values of the scalar dissipation, are more probable when the mixture fraction iso-surface experiences compressive normal strain rates. Comparison of Figs. 6a and d shows that these compressive strains are mainly present when the edge-propagation velocity is negative, i.e. during phase 'E1'. This is also consistent with the results shown in Fig. 4 , which shows that at the initial stage of extinction, the mixture-fraction iso-surface is compressed normally and bulged towards the oxidiser, resulting in high positive mixture-fraction displacement speed. In the second phase of extinction referred to as phase 'E2', the scalar dissipation rate is relaxed and the edge-propagation velocity changes its sign to positive. As shown in Fig. 5b, the positive contribution of stretch terms (equation 4) leads to further expansion of the hole.

The findings in this section suggest that in this lifted flame where the extinction holes might grow as large as three to four times of the jet width [1], the scalar dissipation rate at the boundary of the hole and the strain rate on the surface of the extinction hole are both key parameters. This findings are in contrast to previous modelling assumption of extinction being a sole function of scalar dissipation rates [11].

\subsubsection{Reignition}

The statistical analysis of the local reignition mechanisms is presented in this section. Figure 7 shows the joint PDFs of a) edge-flame propagation velocity and scalar dissipation, b) the edge-flame propagation velocity and the product mass-fraction curvature, c) the product mass-fraction curvature and the scalar dissipation rate and d) normalised flame index and scalar dissipation rates. The black circles are the conditional means. As can be seen in Fig. 7a, the edge-flame propagation velocity is 

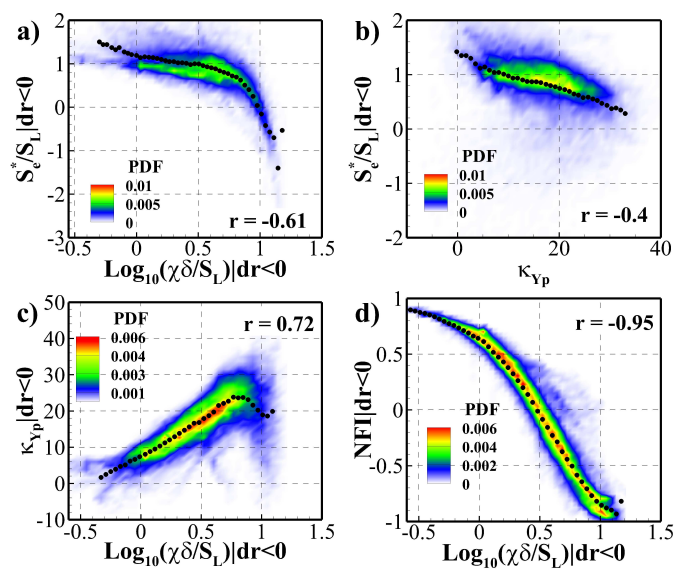

Figure 7: The joint PDFs of a) edge-flame propagation velocity and the scalar dissipation rate, b) edge-flame propagation velocity and the product mass-fraction curvature, c) product mass-fraction curvature and the scalar dissipation rate, and d) NFI and scalar dissipation rates (black circles are conditional means).

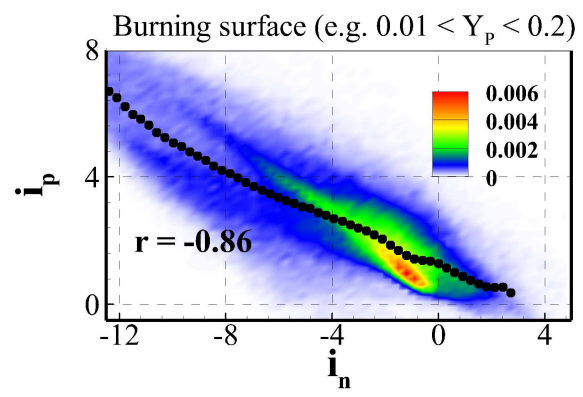

Figure 8: The joint PDF for reigniting holes of thermal diffusion normal to and tangential to mixture fraction iso-surfaces (black circles are conditional means).

mostly positive. Similar to the extinction event, a negative correlation between the edge-propagation velocity and scalar dissipation rate is observed. In addition, Fig. 7b shows that the edge-flame propagation velocity shows a negative correlation with the product mass-fraction curvature. Fig. 7c shows the response of the product mass fraction curvature to the scalar dissipation rate. A strong positive correlation is observed with an almost linear conditional mean with respect to the scalar dissipation rate. To shed more light on the reignition mechanism, the normalised flame index is presented in Fig. 7d. As can be seen, a wide range of combustion modes from premixed to non-premixed flames are present, which is consistent with previous observations in the literature $[15,16]$. This also explains the reason for having almost zero averaged NFI 
observed in Fig. 5e. The results shown in Fig. 7 support the presence of edge flames in the reignition process. However, in order to quantify the significance of edge-flame propagation during reignition, the analysis proposed by Sripakagorn et al. [12] and discussed in section 2 was performed. Figure 8 shows the joint PDF of the normal and on-plane heat diffusion terms, expressed in equation 5. The on-plane thermal diffusion is dominantly positive showing that the heat is transferred from the burned region of the mixture-fraction iso-surface towards the unburned region. This is expected in either edge-flame propagation or engulfment (turbulent folding) scenarios. The normal thermal diffusion obtains both negative and positive values with higher possibility for negative values. As discussed in section $2, i_{n}$ is negative in the edge-flame propagation scenario whereas it is positive in the engulfment scenario. Therefore, Fig. 8 suggests that edge-flame propagation is the main mechanism of reignition. This is in contrast to the previous study of reignition in isotropic three-dimensional decaying turbulence by Sripakagorn et al. [12] where both edge-flame propagation and turbulent folding found to be important during reignition. It also disagrees with the finding of Hawkes et al. [16] in the study of a temporally evolving jet flame, who observed flame-folding to be dominant reignition scenario. A significant difference between the present study and Ref. [16] is the much larger stoichiometric mixture-fraction in Ref. [16], which places the stoichiometric surface with the highly mixed turbulent region, while the present stoichiometric mixture-fraction is much smaller.

The findings show that the reignition is controlled by edge-flame propagation and the free propagation edge-flame model proposed by Boulanger et al. [27] could be a potential model to consider for the reignition process.

Interested readers are strongly encouraged to consult the Fig. S3 in the appendix for a more detailed schematic description of the local extinction and reignition.

\section{Conclusions}

Local extinction and reignition in a turbulent lifted flame were studied using direct numerical simulation (DNS). Separate analyses of extinction and reignition were performed. It was revealed that a high scalar dissipation rate is required for the extinction 
to occur whereas the generated hole can nonetheless grow with significantly lower values of scalar dissipation rate. Two stages of extinction were identified. Initially, holes were created by large structures pushing outwards on the mixture-fraction iso-surfaces. This first phase of extinction was mainly controlled by the normal compressive strain rates on the mixture fraction iso-surface and high scalar dissipation rates. In the second phase of extinction in which the edge-flame propagation velocity is positive, the stretch on the unburned surface of the mixture-fraction iso-surface expands the holes' area. The statistical analysis of the reignition phase shows a dependency of the edgeflame propagation velocity on curvature and scalar dissipation rate which suggests the presence of an edge flame in reignition. Moreover, thermal diffusion analysis on the unburned portion of the mixture-fraction iso-surface reveals that the edge-flame propagation scenario is the dominant reignition mechanism compared with turbulent engulfment ${ }^{3}$.

\section{Acknowledgements}

This work was supported by the Australian Research Council. The research benefited from computational resources provided through the National Computational Merit Allocation Scheme, supported by the Australian Government. The computational facilities supporting this project included the Australian NCI National Facility, Intersect Australia Pty Ltd., Pawsey Supercomputing Centre.

\section{References}

[1] S. Karami, E.R. Hawkes, M. Talei, J.H. Chen, J. Fluid Mech. 777 (2015) 633-689.

[2] G. Amantini, J.H. Frank, B.A.V. Bennett, M.D. Smooke, A. Gomez, Combust. Flame 150 (2007) 292-319.

[3] W.F. Carnell, M.W. Renfro, Combust. Flame 141 (2005) 350-359.

\footnotetext{
${ }^{3}$ However, it is noted that due to the low lifted height in this simulation, the turbulence integral scale is comparable to the flame thickness; if the integral scale were larger, it is possible that engulfment may become dominant
} 
[4] V.R. Katta, K.Y. Hsu, W.M. Roquemore, Proc. Combust. Inst. 27 (1998) 11211129.

[5] R.S. Barlow, J.H. Frank, Proc. Combust. Inst. 27 (1998) 1087-1095.

[6] M. Juddoo, A.R. Masri, Combust. Flame 158 (2011) 902-914.

[7] F. Takahashi, W.J. Schmoll, D.D. Trump, L.P. Goss, Proc. Combust. Inst. 26 (1996) 145-152.

[8] J. Hult, U. Meier, W. Meier, A. Harvey, C.F. Kaminski, Proc. Combust. Inst. 30 (2005) 701-709.

[9] K.M. Lyons, K.A. Watson, C.D. Carter, J.M. Donbar, Combust. Flame 142 (2005) 308-313.

[10] V. Favier, L. Vervisch, Combust. Flame 125 (2001) 788-803.

[11] C. Pantano, J. Fluid Mech. 514 (2004) 231-270.

[12] P. Sripakagorn, S. Mitarai, G. Kosály, H. Pitsch, J. Fluid Mech. 518 (2004) 231259.

[13] R. Knaus, C. Pantano, J. Comput. Phys. 296 (2015) 209-240.

[14] F.N. Egolfopoulos, Int. J. Energ. Res. 24 (2000) 989-1010.

[15] D.O. Lignell, J.H. Chen, H.A. Schmutz, Combust. Flame 158 (2011) 949-963.

[16] E.R. Hawkes, R. Sankaran, J.H. Chen, Proceedings of the Australian Combustion Symposium (2007) 46-49.

[17] C. Pantano, D.I. Pullin, Combust. Flame 137 (2004) 295-305.

[18] S. Karami, E.R. Hawkes, M. Talei, J.H. Chen, Proceedings of the 2014 CTR Summer Program (2015) 137-146. 
[19] S. Karami, E.R. Hawkes, M. Talei, J.H. Chen, Combust. Flame 169 (2016) 110128.

[20] D. Garrido-López, S. Sarkar, Proc. Combust. Inst. 30 (2005) 621 - 628.

[21] K.M. Lyons, K.A. Watson, C.D. Carter, J.M. Donbar, Combust. Sci. Technol. 179 (2007) 1029-1037.

[22] R.L. Gordon, I. Boxx, C. Carter, A. Dreizler, W. Meier, Flow Turbul. Combust. 88 (2012) 503-527.

[23] E. Knudsen, H. Pitsch, Combust. Flame 159 (2012) $242-264$.

[24] S.B. Pope, Int. J. Eng. Sci. 26 (1988) 445-469.

[25] N. Peters, Prog. Energy Combust. Sci. 10 (1984) 319-339.

[26] J. Daou, A. Liñán, Combust. Flame 118 (1999) 479-488.

[27] J. Boulanger, L. Vervisch, J. Reveillon, S. Ghosal, Combust. Flame 134 (2003) $355-368$. 